\title{
Brittle to quasi-brittle transition and crack initiation precursors in crystals with structural Inhomogeneities
}

\author{
S. Papanikolaou ${ }^{1,2^{*}}$ (D) P. Shanthraj ${ }^{3}$, J. Thibault ${ }^{1}$, C. Woodward ${ }^{4}$ and F. Roters ${ }^{3}$
}

\author{
* Correspondence: stefanos. \\ papanikolaou@mail.wvu.edu \\ 'Department of Mechanical \& \\ Aerospace Engineering, West \\ Virginia University, Morgantown, WV \\ 26505, USA \\ ${ }^{2}$ Department of Physics \& \\ Astronomy, West Virginia University, \\ Morgantown, WV 26505, USA \\ Full list of author information is \\ available at the end of the article
}

\begin{abstract}
Crack initiation emerges due to a combination of elasticity, plasticity, and disorder, and it displays strong dependence on the material's microstructural details. The characterization of the structural uncertainty in the original microstructure is typically empirical and systematic characterization protocols are lacking. In this paper, we propose an investigational tool in the form of the curvature of an ellipsoidal notch: As the radius of curvature at the notch increases, there is a dynamic phase transition from notch-induced crack initiation to disorder-induced crack nucleation. We argue that the this transition may unveil the characteristic length scale of structural disorder in the material. We investigate brittle but elastoplastic metals with continuum, microstructural disorder that could originate in a manufacturing process, such as alloying. We perform extensive and realistic simulations, using a phase-field approach coupled to crystal plasticity, where microstructural disorder and notch width are systematically varied. We identify the brittle-to-quasi-brittle transition for various disorder strengths in terms of the damage and stress evolution. Moreover, we investigate precursors to crack initiation that we quantify in terms of the expected stress drops during displacement control loading.
\end{abstract}

Keywords: Microstructural disorder, Crack initiation, Fractals, quasi-brittle fracture, DAMASK, Avalanches

\section{Introduction}

Fracture mechanics of intermetallic materials and other alloys and superalloys remains a critical topic of discussion in the research of mechanical fatigue and fracture. The necessity of intermetallics and superalloys originates in their use for high-temperature applications and components such as gas turbine blades and automobiles (Clemens and Mayer 2013; Stoloff et al. 2000; Kim 1994, and Kim and Dimiduk 1991). Potential applications have spanned from the aerospace industry to electronic devices. While alloying process may lead to excellent mechanical properties like increased oxidation resistance and resistance to creep, it also introduces uncertainty in the form of quenched structural disorder in the material microstructure. For example, ranging over several orders of magnitude, intermetallics display strong variability in strength and other material properties (see also Additional file 1) (Campbell et al. 1999; Dimiduk 1999; Kim 1994, and Kim and Dimiduk 1991). For example, in Titanium Aluminide (TiAl)-based alloys, grain sizes can be as small as $2 \mu \mathrm{m}$ (for fine-grained Ti-rich Ti-Al 
alloy compacts) due to powder metallurgical processes (Vajpai and Ameyama 2013; Lapin 2009) and as large as $800 \mu \mathrm{m}$ due to conventional casting methods and additional alloy compounds like $\mathrm{TiB}_{2}$ (see Additional file 1) (Han et al. 2015; Hu 2001). Similar to TiAl, Nickel Aluminide (NiAl)-based alloys and Inconel express similar microstructural length scale characteristics (see also Additional file 1) (Zhu et al. 2018; Herbold et al. 2011). Due to the very presence of this structural disorder, alloys display stochastic fracture characteristics, especially when cracks can be labeled "short". The range of fatigue growth rates for short cracks may extend well beyond 4 orders of magnitude (approximately $10^{-11}$ to $10^{-6} \mathrm{~m} /$ cycle) (see Additional file 1 ) (Ritchie and Peters 2001). It is natural to interpret these wide-ranging short-crack-growth rates as being an outcome of alloying disorder and thus, may be associated to avalanche precursors in fatigue, analogous to analogous phenomena in various complex systems (Papanikolaou et al. 2017). In this paper, we discuss in detail how such stochastic fracture, and its avalanche precursors, can emerge in a model material with relatively realistic parameters and we explore the possibility of characterizing and quantifying the structural disorder in a statistical manner by locating a naturally-occuring brittle-to-quasi-brittle dynamical transition.

Microstructural heterogeneity is an unavoidable effect that originates in the multiple processes used during manufacturing of alloys. In analogy to other disordered manybody systems, it is natural to envision that this material heterogeneity gives rise to abrupt crack-growth events, especially at short length scales compared to the microstructural heterogeneity. Given the limited statistical sampling of short crack growth in mechanical components, it is imperative to clarify ways and protocols that can capture, controllably and systematically, this precursor behavior to fracture. We envision an experimental protocol for identifying and controlling the onset of such precursor events. In order to pursue such a protocol, we utilize a model material which could be considered a simplification of an intermetallic alloy. It is well recognized that alloys such as NiAl may contain multiphase variability at a length scale of few microns as a result of alloying processes (Wang et al. 2016; Herbold et al. 2011, and Lay and Yavari 1996). We model this structural disorder as a continuum field that influences the critical strain energy release rate in the material. We also model macroscale fracture of such alloys as brittle (room temperature), while at the micron scale we consider plastically deforming processes. In this way, crack growth in brittle fracture competes with crystal plasticity at the microscale.

The statistical effects of material heterogeneity in short-crack growth for alloys and superalloys have remained elusive: While it is well known how alloying processes produce several precipitates such as $\gamma^{\prime}$ and $\gamma^{\prime \prime}$ phases (Tian et al. 2014), it is unknown how they influence the probability of large or small crack growth "bursts" as fatigue cycles proceed. Motivated by basic elastic fracture mechanisms (Zehnder 2012), it is natural to refer to the so-called "quasi-brittle" fracture, which is motivated by large-scale fracture of disordered specimens, such as concrete. Early experiments of fracture on concrete did not obey the criteria that characterize Linear Elastic Fracture Mechanics (LEFM) (Walsh 1972) with the ultimate conclusion (Grote et al. 2001; Schlangen 1993; Schlangen and Van Mier 1992, and Bazant and Pfeiffer 1985) being that a nonlinear approach for predicting fracture in these materials is essential to understanding and characterizing this deviant fracture behavior (Schlangen and Garboczi 1997; Bažant et al. 
1990, and Bažant and Oh 1983). While material heterogeneity of rock, concrete, wood and ice are considered at large length scales on the order of a few millimeters (Skarynski and Tejchman 2010), analogous results can be considered mutatis mutandis at smaller length scale structural disorder in alloys and superalloys.

Quasi-brittle materials contain inhomogeneities that cannot be considered negligible when compared to the structure size (Bažant et al. 2009). As a result, the fracture strength of a structure may greatly depend on its size. This phenomenon, typically referred to as size effect, has been observed in many materials from bone (Evans 1973) to concrete (Bažant 1984). However, alloys and superalloys display similar fracture characteristics at much smaller scales, such as a few microns. If both concrete and alloys share similar fracture characteristics, then it is natural to investigate whether scaling relations for quasi-brittle materials may be applied to alloy-relevant smaller length scales. For example, in Refs. [(Bazant and Planas 1998, Bažant 1996, and Bažant and Li 1996)], it was suggested that quasi-brittle materials can be characterized using a scaling relation for which fracture strength could be predicted,

$$
\sigma_{F}=B f_{t}^{\prime}\left(1+\frac{a}{a_{0}}\right)^{-1 / 2}
$$

This scaling relation can be derived by asymptotic matching of the fracture stress as a function of size, represented in Eq. (1) where $\sigma_{F}$ is the fracture strength, $B$ is a dimensionless constant, $f_{t}^{\prime}$ is the tensile strength, $a_{0}$ is a constant referred to as the transitional size, and $a$ is the characteristic size of the structure (Bazant 1985; Bažant 1984). Is such a scaling relation valid for superalloys and intermetallics?

Our methodology is based on the possible and viable ways to classify the short crack growth regime by developing an experimental protocol that may cause a transition in fracture behavior. Due to advances in microfabrication techniques, namely focused ion beam (FIB), it is now possible to machine precise-dimensioned notches at the micron and submicron scale to a sub-10 $\mathrm{nm}$ resolution (Ochiai et al. 2010; Tseng 2004). It is realistic to envision high-throughput experiments where one machines and tests various notch curvatures in order to observe the variety of patterns in fracture behavior. We pursue the direction of modifying the curvature of a fixed-depth notch where it is naturally expected that the notch will influence fracture only after it becomes sharp enough, defining a quasi-brittle-to-brittle transition as a function of notch curvature. At which length scale does the material's structural disorder cause a transition from brittle to quasi-brittle fracture behavior? In this paper, we attempt to find and characterize this transition as a function of the notch curvature.

Crack initiation from a notch is commonly believed to be controlled by pure elasticity. However, heterogeneous materials contain inhomogeneities that may contribute as much to fracture as a notch. If the notch curvature decreases, the stress concentration at the notch root will decrease and, as a result, stress shall redistribute within the bulk of the material. Notched, brittle and homogeneous materials have been intensely studied (Williams 1952), while the effects of a notched geometry in heterogeneous materials has been of more recent focus, with studies as function of the notch length and opening angle (Carpinteri 1987; Carpinteri et al. 1996; Dunn et al. 1997a; Dunn et al. 1997b) or in simplified models of fracture (Alava et al. 2009). However, the interplay between 
structural disorder and notch geometry has not been studied, especially in a micromechanical model that properly captures elasticity, plasticity and damage as mechanical load is applied on a sample.

In this work, we study a continuum micromechanical model of a structurally disordered material. We simulate a transition from notch-driven crack initiation, characteristic of LEFM, to bulk crack nucleation, characteristic of quasi-brittle fracture, using a model material that resembles a brittle material that may plastically deform at the microscale. We use an integrated spectral phase field approach coupled constitutively to elastoplasticity through the Düsseldorf Advanced MAterial Simulation Kit (DAMASK) software (Roters et al. 2019). Elasticity and crystal plasticity are modeled through typical constitutive laws that take into account grain orientation, crystalline structure, and slip systems (Asaro 1983). Through the application of this multiscale, micromechanical analysis, the microstructure can be linked to the macroscopic response. In this paper, we perform extensive simulations of a notched specimen with dimensions: $L_{x} x L_{y} \times L_{z}$ $\sim(8 \mu \mathrm{m} x \mathrm{~L} \mu \mathrm{m} \times 1024 \mu \mathrm{m})$. The test samples have an assumed length scale of $1 \mathrm{~mm}$ per 256 unit representative volumes (each unit corresponds to $\sim 4 \mu m$ ). $L_{x}$ and $L_{z}$ are held constant at $8 \mu \mathrm{m}$ and $1.0 \mathrm{~mm}$, respectively, and $L_{y}$ is varied from $0.25 \mathrm{~mm}$ to 1.0 $\mathrm{mm}$. This particular resolution is chosen for purely numerical reasons, so that a sampling rate of 20 initial disorder realizations per parameter combination becomes possible. For this system size, fracture is completed in less than $24 \mathrm{~h}$ in 4 Intel Xeon E7 computer cores. The ellipsoidal notch is placed along the $y$-axis at the edge of the specimen, and the major axis, $a=L_{y} / 8=64 \mu \mathrm{m}$, is held constant throughout this study.

In order to observe the transition from notch-driven crack initiation to bulk-induced crack nucleation, the notch width, $W$, is varied from $16 \mu \mathrm{m}$ to $0.5 \mathrm{~mm}$, by decreasing the radius of curvature. In order to modify in a generic fashion the microstructural propensity to fracture and introduce a particular disorder length scale, we consider the quenched stochastic contribution of the local critical strain energy release rate $G_{C}$ that controls the rate of local damage accumulation, and we define the variance ratio of this disorder parameter as $R_{G}=\delta G_{C} /\left\langle G_{C}\right\rangle$. For the generation of spatially continuum and stochastic disorder, we use the Weierstrass-Mandelbrot function (Shanthraj et al. 2011) in which the fractal dimension, $D$, is set at either 2.85 or 2.995 . The fractal dimension specifies the "smoothness" of the continuous W-M function in space and if the value of $D$ is larger than 2, then the function is a fractal. For our purposes, local sharpness of the function becomes practically significant when $\mathrm{D}>2.6$. The $\mathrm{W}-\mathrm{M}$ function is used to impose a disorder distribution in the value of the material property, $G_{C}$, that controls the damage evolution in the phase-field model. For every combination of $\left(R_{G}, W\right)$, we consider high-throughput simulations (20 realizations per case) in order to statistically sample microstructure fluctuations.

In what follows, we describe the main outcomes of our study. In Section 2, we explain the continuum model, its disorder distribution generation, and the overall phase field approach. In Section 3, we discuss the key simulation results and in particular, the features of the dynamical transition that is induced by a notch with variable curvature. Finally, in Section 4, we present our conclusions about the importance of this work and possible future directions. In Section 5, we present the abbreviations used in the manuscript, in Section 6 we display some declarations, and the manuscript ends with a list of references. 


\section{The model: material disorder, phase-field damage and crystal plasticity}

In this work, we use a phase field model for a thorough continuum simulation of elasticity, plasticity and damage in three-dimensional mechanics. This model is implemented through an open-source software (Roters et al. 2019). that utilizes a continuum phase field model for material deformation and damage evolution within the sample. For details in the open-source numerical software, it may seem useful to consult Ref. (Roters et al. 2019). In this work, we use a spectral method to solve for the elastic and phasefield equations, using Fast Fourier transforms (FFT). Spectral methods are very stable numerically, especially for handling structural disorder. However, this spectral character requires a rectangular-gridded mesh and periodic boundary conditions in all spatial directions. In the horizontal direction, we apply a layer of air (as an elastic material with infinitesimal elastic coefficients) in contact with the notched surface of thickness $64 \mu \mathrm{m}$. The layer of air is in contact to the specimen from both left and right directions. The thin layer of air has no effect on the mechanics of the specimen, given that deformation fluctuations in the y-direction are small in comparison to the air layer thickness. Given that loading is in the z-direction, the deformation in the y-direction is infinitesimal, so modeling in the $y$-direction is consistent. In the vertical (and z) directions, the sample is periodic, thus is assumed practically bulk/infinite in these directions.

\section{Phase field approach}

In this work, we utilize a phase field approach (Aranson et al. 2000) where cracks are modeled by a phase with order parameter $\phi=0$, while the intact material has $\phi=1$. The field $\phi$ couples proportionally to the elastic coefficients of the material. (see also Additional file 1). The phase field enters an appropriately defined free energy:

$$
\psi=\psi_{E}+\psi_{D}+\psi_{P}
$$

where $\psi_{E}, \psi_{D}$, and $\psi_{P}$ are the elastic, damage, and plastic free energy contributions, respectively.

The elastic energy is decomposed in a direct multiplicative manner to the simplest damage contribution $\left(\phi^{2}\right)$ and a pure distortion-dependent contribution:

$$
\psi_{E}=\phi^{2} \widetilde{\psi_{E}}\left(\nabla X, F_{p}\right)
$$

At the undamaged state, the sole energy contribution to the sample is elastic free energy. The stored elastic energy density is dependent on the product of the dissipation potential, $X$, and the plastic deformation tensor $F_{p}$. As loading progresses, the strain energy release rate, $G$, reaches a critical point where the elastic free energy in the disordered distribution, locally, is converted to damage. The critical strain energy release rate, $G_{c}$, is how the model determines if damage has occurred. Locally, these areas will only exhibit damage and plastic free energy (Shanthraj et al. 2016).

Damage accumulation is determined by the grid variables as the critical strain energy release rate values are assigned. As strain increases, all energy contributions are tracked at each incrementally-increasing strain-steps. When the damage free energy is nonzero,

$$
\psi_{D}=1 / 2 G_{c} l|\nabla \phi|^{2}+G_{c} / l_{l}(1-\phi)^{m}+I_{[0,1]}(\phi)
$$


where $G_{c}$ and $l$ are material constants and $m$ is the order of the phase field potential (typically $m=1$ or 2 ). $G_{c}$ controls the free energy contribution necessary for damage accumulation to occur, and $l$ is the resolution length scale which corresponds to a representative volume element $(R V E)$ of the material (Shanthraj et al. 2016). In our simulations, we consider a fixed resolution length $1=4$ mesh units, corresponding to $16 \mu \mathrm{m}$, given our considered sample dimensions. As damage evolves, there is also permanent deformation that contributes to the plastic part of the free energy.

Quantifying the plastic free energy requires an indirect approach with the help of several constitutive laws. The phase field equation for the plastic deformation tensor gives,

$$
\partial F_{p} \psi=-\phi^{2} S F^{-T}
$$

which we may reduce into the familiar slip system equation for crystalline materials,

$$
-\partial F_{p} \psi \cdot F_{p}=\sum_{\alpha} \partial \gamma^{\alpha} \psi \cdot \gamma^{\alpha}
$$

where the sum is acoss slip systems and,

$$
\partial \gamma^{\alpha} \psi=-\phi^{2} S \cdot\left(s^{\alpha} \otimes n^{\alpha}\right)=\tau^{\alpha}
$$

where $\tau^{\alpha}$ refers to the shear stress along slip system $a$ and $S$ refers to the 2nd PiolaKirchoff stress tensor.

At this stage, several constitutive plasticity laws are required: For estimating the plastic deformation tensor and its rate, we iteratively solve: ' $F_{p}=L_{p} F_{p} . L_{p}$ is a function of the slip rate along each slip system $\gamma^{\alpha}$,

$$
L_{p}=\sum_{\alpha} \gamma^{\alpha} s^{\alpha} \otimes n^{\alpha}
$$

where $s$ and $n$ being the unit vectors along the slip direction and slip plane normal, respectively, and $\alpha$ is the index of a slip system. We consider all 12 FCC slip systems which may become active in our single crystal with fixed crystalline orientation. The slip rate $\gamma^{\alpha}$ is given by the basic phenomenological crystal plasticity constitutive equation (Asaro and Lubarda 2006),

$$
\gamma^{\alpha}=\gamma_{o}\left|\frac{\tau^{\alpha}}{g^{\alpha}}\right|^{n} \operatorname{sgn}\left(\tau^{\alpha}\right)
$$

where $\gamma_{o}$ is the reference shear rate, $\tau^{\alpha}=S \cdot\left(s^{\alpha} \otimes n^{\alpha}\right), n$ is the stress exponent and $g^{\alpha}$ is the slip resistance stress for a slip system $\alpha$. In this formula, the hardening rate is provided by another phenomenological crystal plasticity constitutive law:

$$
g^{a}=\sum_{\beta=1}^{12} \gamma^{\beta} h_{0}\left|1-\frac{g^{\beta}}{g_{\infty}^{\alpha}}\right|^{w} \operatorname{sgn}\left(1-\frac{g^{\beta}}{g_{\infty}^{\alpha}}\right) h_{\alpha \beta}
$$

where $w$ and $h_{0}$ are hardening parameters, $g_{\infty}^{\alpha}$ is the saturation stress, and $h_{\alpha \beta}$ is the hardening matrix which captures the interactions between different slip systems (Shanthraj et al. 2016).

\section{Heterogeneous microstructure generation}

We identify disorder in terms of the variability for the critical strain energy release rate $G_{c}$. We assume throughout that $G_{c}$ displays local, quenched and continuous fluctuations, as it would naturally happen in a multi-phased disordered metal alloy or a 
fatigued specimen. Namely, we assume that $\delta G_{c} \rightarrow\left\langle G_{c}\right\rangle+\Delta G_{c}(y, z)$ and the local fluctuations $\Delta G_{c}(y, z)$ are given in space through a W-M function with an autocorrelation length scale of $160 \mu \mathrm{m}$ at a given fractal dimension $D$ (see Fig. 3g). The autocorrelation length scale corresponds to the spatial distance where the W-F function fluctuations are practically decorrelated. The autocorrelation length is ultimately decided by the W$\mathrm{M}$ function's parameter combination. The W-F's autocorrelation length scale is chosen so that a transition due to the notch width becomes visible for the parameter ranges that are chosen in this work and remains fixed across all simulations. The experimentally relevant parameter that is tuned in these simulations is the degree of relative disorder fluctuations, or in other words the width of the distribution of $G_{c}$ with respect to the average critical strain energy release rate. If we define the standard deviation of the distribution as $\delta G_{c}$, then the important quantity to investigate would be $R_{G}=\delta G_{c} /\left\langle G_{c}\right\rangle$. In the next section, we discuss how this phase field model in a realistic elastoplastic environment can cause crack initiation and fracture at various notch widths and disorder strengths, keeping $\left\langle G_{c}\right\rangle$ fixed but changing the disorder ratio $R_{G}$.

The W-M function allows for modeling of structural disorder using microscopic roughness in the energy required for local damage increase. More specifically (Weierstrass 1895; Mandelbrot 1979).

$$
\begin{aligned}
\Delta G_{c}(y, z)= & L\left(\frac{G}{L}\right)^{D-2}\left(\frac{\ln \gamma}{M}\right)^{1 / 2} \sum_{m=1}^{M} \sum_{m=0}^{n_{\max }} \gamma^{(D-3) n} \\
& \times\left\{\cos \left(\phi_{m, n}\right)-\cos \left[\frac{2 \pi \gamma^{n}\left(y^{2}+z^{2}\right)^{1 / 2}}{L} \times \cos \left(\tan ^{-1}\left(\frac{z}{y}\right)-\frac{\pi m}{M}\right)+\phi_{m, n}\right]\right\}
\end{aligned}
$$

Within the W-M function, the parameter tuned throughout the paper is $\mathrm{G}$, which is chosen so that $R_{G}$ is the control parameter. All other parameters $\left(\gamma, M, L, n_{\max }\right)$ remain the same $(3,5,256,4)$ across all simulations of this work and $\phi_{\mathrm{m}, \mathrm{n}}$ are flat random in $[0, \pi]$. Their choice controls the overall form of the W-M function (Mandelbrot et al. 1984). The fractal dimension, D, exhibit a strong influence on the spatial fluctuations in the energy and stochastic characteristics (Mandelbrot et al. 1984; Shanthraj et al. 2011, and Carney and Mecholsky 2013). The physical meaning of the other (than G, D) parameters that help manipulate the fracture behavior, is: $L$ is the sample length, $\gamma$ is a scaling parameter, $M$ is the superposed ridges used to construct the surface for its roughness, $\phi_{\mathrm{m}, \mathrm{n}}$ is a random phase with $m$ and $n\left(n_{\max }\right)$ defining the ridge and frequency index, and $\mathrm{y}[\mathrm{m}]$ and $\mathrm{z}[\mathrm{m}]$ correspond to locations in space.

The choice of the W-M function for the generation of structural disorder serves the purpose of making the high-throughput simulations of this work efficient and well controlled. The W-M function satisfies the features of continuity, smoothness, as well as jerkiness, so that the numerical spectral solver achieves convergence in reasonable simulation time intervals. Namely, given that W-M is always smooth at the resolution scale for the given parameters, DAMASK's FFT spectral convergence is always achieved. Moreover, the large fractality $(\mathrm{D} \rightarrow 3)$ guarantees very sharp local features in the sample. W-M functions are efficiently calculated and they are simply controlled by just few parameters. 
Nevertheless, the choice of the W-F function for the generation of structural disorder fluctuations is not unique: It is worth noting that another plausible choice for the implementation of structural disorder across a sample is the choice of locally random parameters (from any stochastic distribution, e.g. Weibull or Poisson) (Alava et al. 2009) which should be eventually smoothened for the purposes of this implementation (if the disorder is not spatially smoothened, the current simulation technique has been noticed to display very inefficient convergence rates). For the choices of this work, the process of smoothening introduces additional spatial correlations and artifacts, that are not well controlled or understood. For these reasons, we believe that the W-M function is ideally suited to the structural disorder implementation in the DAMASK model.

\section{Simulation results}

We investigate a typical mode-I crack initiation scenario, where a thin film of dimensions $(8 \mu \mathrm{m} \times \mathrm{L} \mu \mathrm{m} \times 1024 \mu \mathrm{m})$ is loaded along the long $z$-direction at a displacement controlled loading rate of $10^{-1} / \mathrm{s}$. The sample also contains an elliptical notch at the location where $z$ is centered (see Fig. 1). The objective of the simulations is to identify characteristic effects of a stochastic damage distribution and how it may contribute to the fracture mechanics of the specimen. When crack initiation occurs, the natural outcome is to occur at the notch for brittle materials, given that the stress magnitude is far higher at the notch tip than any place in the bulk, allowing failure to occur at this point. However, the induced disorder distribution in the specimen could allow for crack nucleation away from the tip of the notch. The material parameters of the model are shown in Table 1 and discussed in the Additional file 1.

Simulations (not shown) were conducted at several fractal dimension values to determine a particularly chosen value for $D(=2.85)$ so that the fluctuations in the critical strain energy release rate would affect the crack nucleation and propagation behavior. We will be only studying two cases throughout this work $(D=2.85$ and 2.995). The choice of the fractal dimension was promoted by the condition that sharp structuraldisorder features in the material's bulk generated higher Von Mises stresses than stresses generated due to discretization issues near the sample boundaries (see also Additional file 1). The fractal dimension is important in that crack initiation is not caused by discretization artifacts near the sample boundaries, which naturally lead to artificial stress concentrations. If the fractal dimension is too low $(D \rightarrow 2)$, then crack initiation takes place due to the fact that the notch is resolved into the simulation's cubic grid. (see also Additional file 1 for more details). We investigate a range of variances $\delta G_{c}$, so that the ratio $\mathrm{R}_{\mathrm{G}}$ is sampled thoroughly in the physically relevant region $[0,1]$.

The competition between the spatial fluctuations of $\mathrm{G}$ (due to spatially varying $\delta G_{c}$ ) and the length scale associated with the notch, becomes evident in the increase of the notch curvature. Figure 2 shows the damage (where 1 is undamaged and 0 is fully damaged) and stress $(\mathrm{Pa})$ distributions for initial crack widths of $16 \mu \mathrm{m}, 128 \mu \mathrm{m}$, and $0.5 \mathrm{~mm}$. In this case, there is a transition from notch-driven crack initiation to quasi-brittle crack nucleation in the material's bulk.

\section{Crack initiation for fixed notch width as function of increasing local critical strain energy release rate's variance}

Analogously to other works (Bazant and Planas 1998; Alava et al. 2008), the increase of disorder leads to the onset of quasi-brittle behavior, namely crack nucleation initiates 


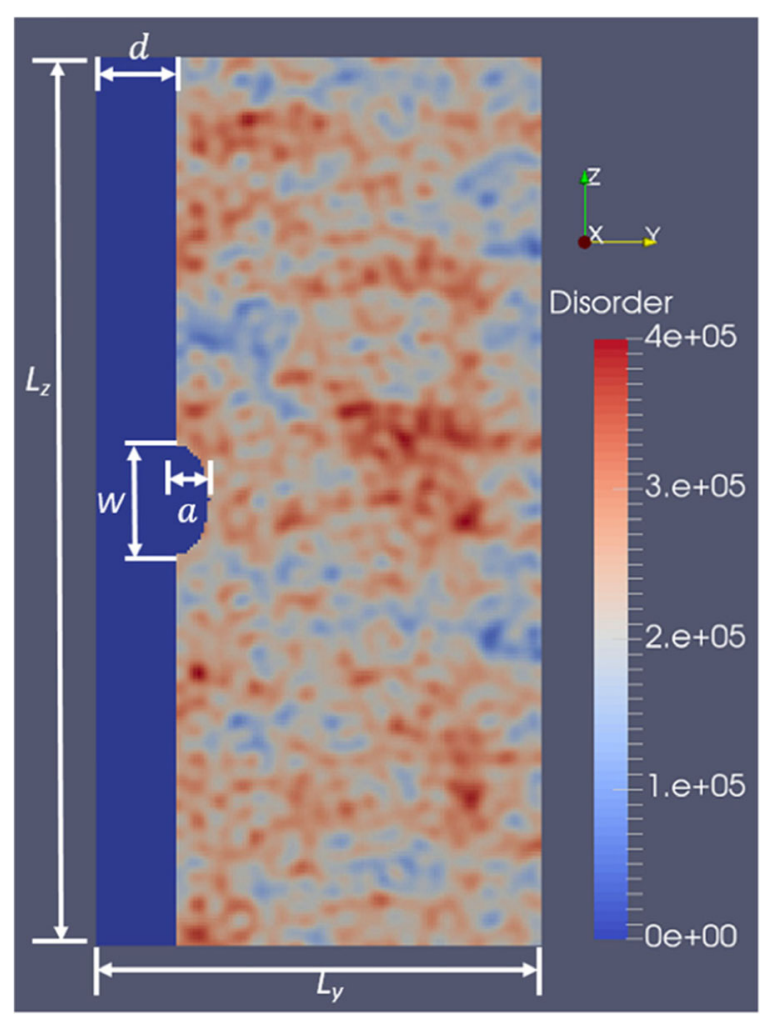

Fig. 1 Model Geometry \& Disorder: The thickness of the sample, $L_{x}=8 \mu \mathrm{m}$, the width of the sample $L_{z}=1.0$ $\mathrm{mm}$, the length of the sample, $L_{y}=0.5 \mathrm{~mm}$. The layer of air has thickness $d=96 \mu \mathrm{m}$, and the notch is an ellipse with constant major axis of $\mathrm{a}=64 \mu \mathrm{m}$.. Here, the notch width $\mathrm{W}$ is $128 \mu \mathrm{m}$, but it is varied throughout this work. Similarly, the disorder ratio in this example is $\mathrm{R}_{\mathrm{G}}=0.2$

at bulk locations, where the weakest sites exist. Here, we investigate this phenomenon in the presence of a notch, and in the context of our continuum phase-field model and through the use of the W-M function towards the disorder distribution generation.

Figure 3 depicts a progression of increasing $R_{G}$. As $R_{G} \rightarrow 0$, the fluctuations in the critical $J$-integral value, $J_{I O}$ will be relatively small leading to elastic response. As $R_{G} \rightarrow 1$, the variation in the critical strain energy or material fracture toughness will overcome the elastic stresses originating from the notch curvature in inducing damage.

Table 1 Phase parameters used in the simulations for the model material (Tikhovskiy et al. 2007)

\begin{tabular}{lll}
\hline Property & Value (Model Material) & Units \\
\hline$C_{11}$ & 106.75 & $\mathrm{GPa}$ \\
$C_{12}$ & 60.41 & $\mathrm{GPa}$ \\
$C_{44}$ & 28.34 & $\mathrm{GPa}$ \\
$\gamma_{0}$ & $1.0 \times 10^{-3}$ & $\mathrm{~s}^{-1}$ \\
$n$ & 20 & \\
$g_{0}$ & 31 & $\mathrm{MPa}$ \\
$g_{\infty}$ & 63 & $\mathrm{MPa}$ \\
$a$ & 2.25 & \\
$h_{0}$ & 75 & $\mathrm{MPa}$ \\
$h_{\alpha \beta}$ & 0 & \\
\hline
\end{tabular}




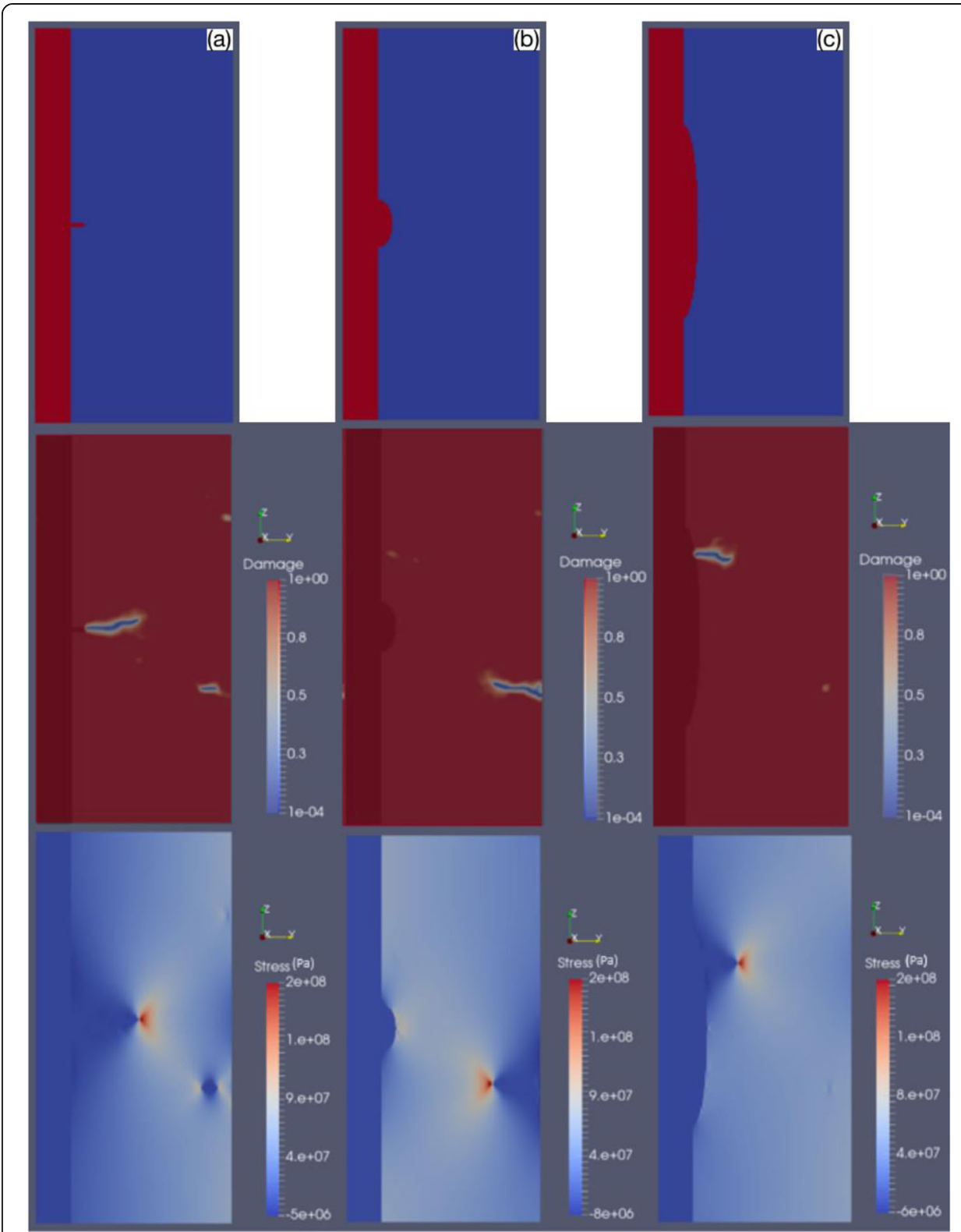

Fig. 2 Effect of increasing notch width on crack nucleation: Simulations of system size of $L_{x}=8 \mu m, L_{y}=0.5$ $\mathrm{mm}$, and $\mathrm{L}_{z}=1.0 \mathrm{~mm}$ where $\mathrm{D}=2.85, \mathrm{R}_{\mathrm{G}}=0.8$, and (a) has a $\mathrm{W}$ of $16 \mu \mathrm{m}$ (b) has a $\mathrm{W}$ of $128 \mu \mathrm{m}$ (c) has a $\mathrm{W}$ of $0.5 \mathrm{~mm}$ where the texture, damage and stress (along the loading direction $\sigma_{\mathrm{zz}}$ ) distributions are displayed

Physically, the $R_{G}$ value allows one to control the variation in the toughness of the material. We choose the mean critical strain energy parameter, $G_{c}$, of the material to be a value of $200 \mathrm{~kJ} / \mathrm{m}^{2}$, rendering the sample quite brittle. As Fig. 3 shows, our simulations are performed in such a way that the average $G_{c}$ remains relatively constant as we modify the variance of $G_{c}$. In Fig. 3, the distribution for $R_{G}=0.8$ and $W=0.125 \mathrm{~mm}$ exhibits a much larger variation in the range with parts of the distribution having higher values of critical strain energy release rate, indicated by red, versus lower values of critical strain energy release rate, indicated by shades of blue. Furthermore, panel (e) refers to the histogram of the critical strain energy release rate. 

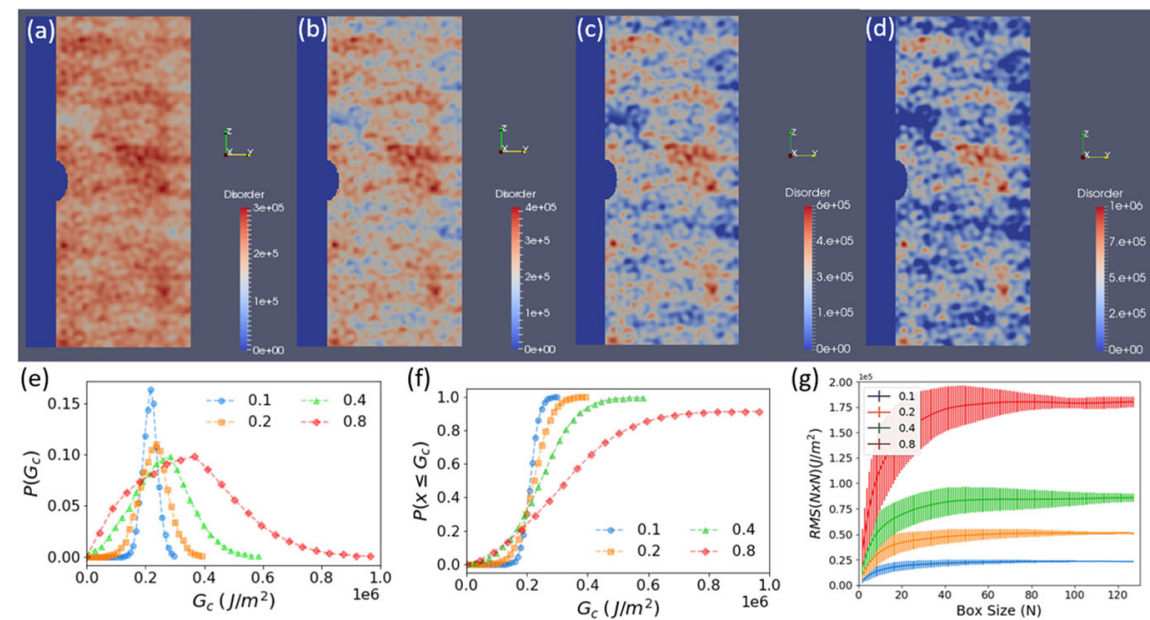

Fig. 3 Disorder distribution for local fracture tendency and spatial profiles: The progression of the disorder ratio, $R_{G}$, for $W=0.125 \mathrm{~mm}$ with a system size of $L_{x}=8 \mu \mathrm{m}, L_{y}=0.5 \mathrm{~mm}$, and $L_{z}=1.0 \mathrm{~mm}$ where (a) has a disorder ratio of $0.1,(\mathbf{b})$ has a disorder ratio of 0.2 , (c) has a disorder ratio of 0.4 , (d) has a disorder ratio of $0.8,(\mathbf{e})$ and (f) are the probability density function (pdf) and cumulative distribution function (cdf), respectively, of the fluctuating phase field energy parameter of each node vs. the phase field energy parameter, and $(\mathbf{g})$ is the autocorrelation lengthscale of the W-M function. The saturation of the autocorrelation function may be thought as the disorder correlation length (Alava et al. 2009)

The main parameters of the model are the ratio $R_{G}$ and the radius of curvature at the ellipse notch's horizontal location. Here, the notch has major, minor axis as a and w/2 (see Fig. 1), which lead to a curvature at the notch tip $\kappa_{\text {notch }}=4 \frac{a}{w^{2}}$.

In the presence of disorder, the crack initiation occurs typically at the initial crack tip. In Fig. 4, crack initiation as function of disorder is shown: when the $R_{G}$ value is 0.05 (panel a), while the crack initiated at another location away from the notch tip for $R_{G}$ of 0.2 and 0.8 from (panels b and c), respectively. It can be seen that the crack propagation was affected by the increased variance in the critical strain energy release rate. At the lower disorder ratio distributions, the crack is rather straight and remains so as it propagates through the specimen. However, as the disorder ratio increases, the observed trend shows that crack nucleation occurs away from the initial notch. Then, at even higher disorder strengths, multiple cracks begin to nucleate across the entire specimen. For the cases where $R_{G}<0.2$, we observe that the crack propagates at the notch, but as it continues through the specimen, the crack's path is altered, moving through areas of lower critical strain energy release rate as a result of the introduced disorder. There is a transition in the behavior: At low disorder ratio $R_{G}$, the specimens' exhibit behavior that follows brittle fracture mechanics, nucleating at the notch tip, but, at higher disorder strengths, notchdriven fracture transitions from brittle to a quasi-brittle, disorder-driven fracture with nucleation behavior that is influenced by crystal plasticity.

As we focus closer on this phenomenon with respect to disorder, we examine the damage and stress distributions. However, it is pertinent to this discussion to look at the time of the damage accumulation that causes crack initiation by considering various snapshots during the loading of the specimen for the case of $W=0.125 \mathrm{~mm}$ and $R_{G}=0.8$.

Figure 5 exhibits the fracture behavior as tensile load increases. The undamaged phase, (a), is used as a comparison with the progression of damage and stress 


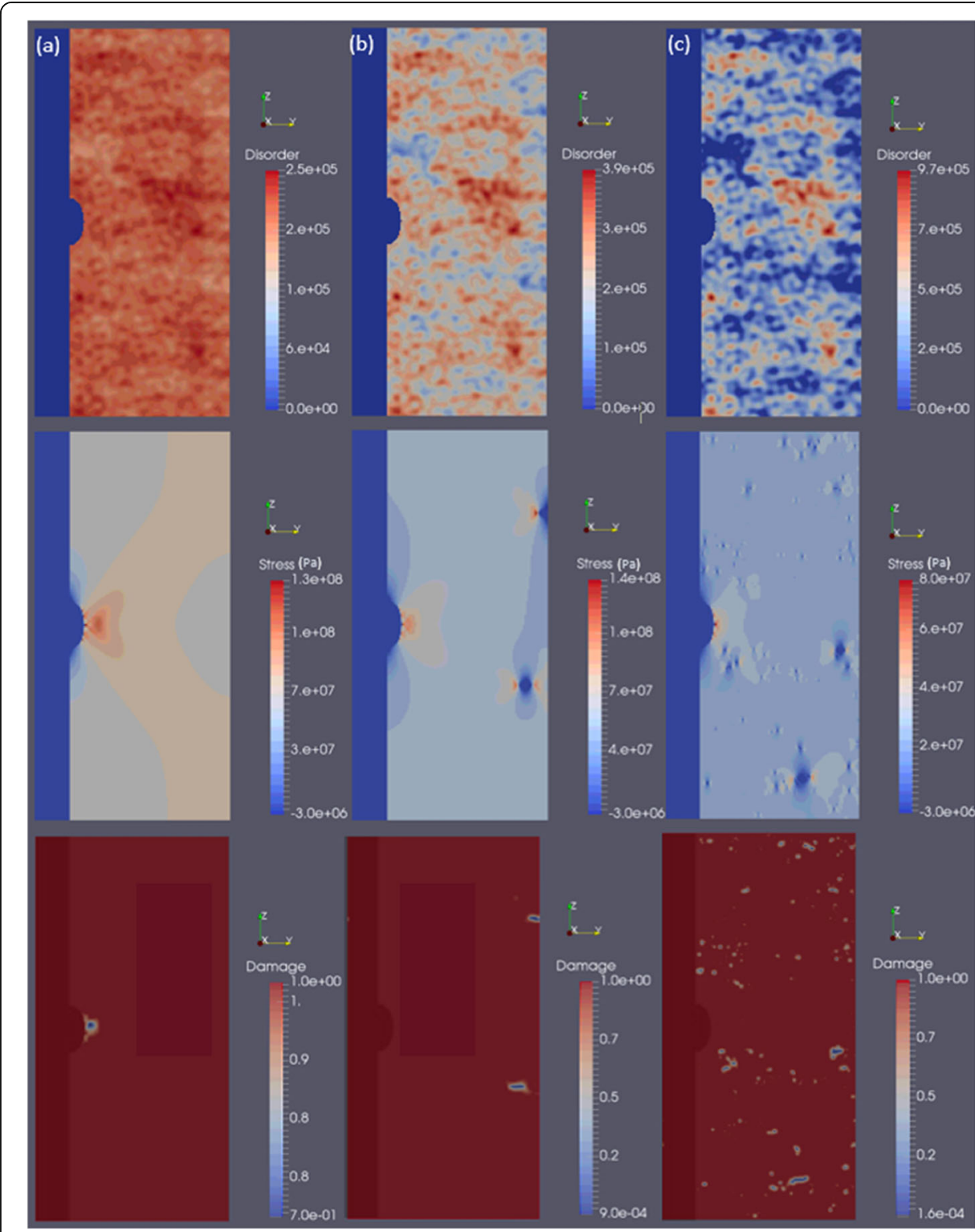

Fig. 4 Effect of increasing disorder strength on crack nucleation: Simulations of system size of $L_{x}=8 \mu m$, $L_{y}=0.5 \mathrm{~mm}$, and $L_{z}=1.0 \mathrm{~mm}$ where $D=2.995, W=0.125 \mathrm{~mm}$, and (a) has a $R_{G}$ of 0.05 (b) has a $R_{G}$ of 0.2 (c) has a $R_{G}$ of 0.8 where the disorder, stress and damage distributions are displayed (top, middle, bottom respectively)

distributions. As load increases, the next phase, (b), reveals that damage has occurred in the bulk of the sample and the stress has increased at the notch tip. Stress is beginning to increase at multiple locations throughout the sample where the critical strain energy release rate is lower compared to the mean. As loading further continues, damage accumulates and crack initiation occurs, but crack initiation occurs at multiple locations on the sample, as can be seen in panels (c), (d), and (e). There are several factors that determine the character of the $\operatorname{crack}(\mathrm{s})$ that eventually propagate(s) through the specimen. Crack features are determined by material properties like critical strain energy release rate, stress distribution at the current load step, statistical modelling, and proximity to other inclusions. 

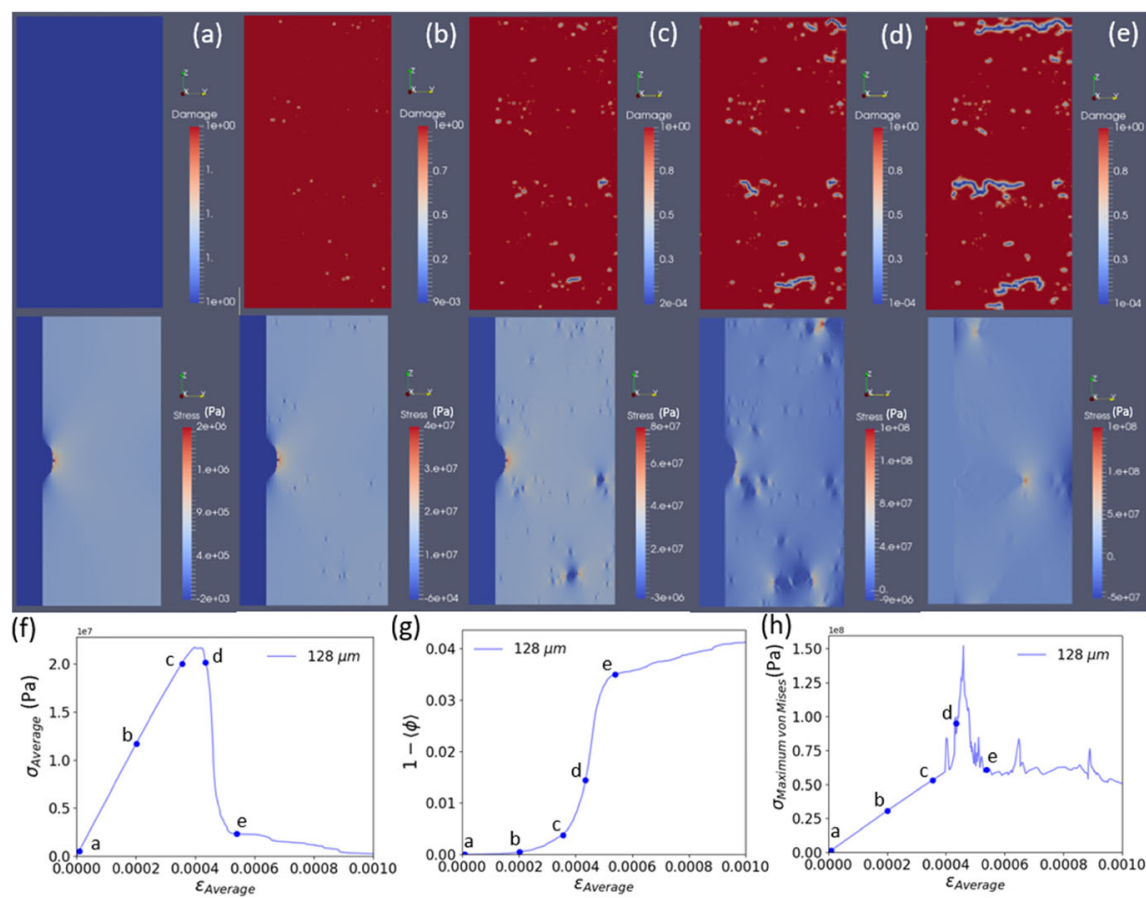

Fig. 5 Stress and damage progression in a disordered, notched, crystalline sample: Simulations of system size of $L_{x}=8 \mu \mathrm{m}, L_{y}=0.5 \mathrm{~mm}$, and $L_{z}=1.0 \mathrm{~mm}$ where $D=2.995, W=0.125 \mathrm{~mm}$, and $R_{G}=0.8$ where $(\mathbf{a})$ is the undamaged phase, (b) is the early damage phase, (c) is the late damage accumulation phase, (d) is the multiple crack nucleation event, and (e) is the crack propagation where the damage and stress distributions and are displayed (top, middle), respectively, and (f), (g), and (h) are the average stress (Pa) vs. strain, average damage vs. strain, maximum von Mises stress ( $\mathrm{Pa}$ ) vs. strain (bottom) respectively, where the five locations are identified on the curves

Width of notch and its effects on crack initiation and propagation

For our simulations, we analyze the behavior under Mode I fracture where tension or compression is used to initiate a crack. LEFM is used to approximate the stresses at the crack tip. However, it is well recognized that this model is inaccurate in a limited region of the Fracture Process Zone (FPZ) (Bažant and Yu 2004). The small scale yielding model for the size of this region can be approximated so that the stresses outside the process zone are accurate. For an ellipsoidal crack shape with initial crack length $a$, the stress intensity factor at the tip can be approximated by $K_{I}=\sigma_{\infty} \sqrt{\pi a}$ (where $\sigma_{\infty}$ is the tensile stress that the sample is subjected to). As the length of the crack increases, the stress intensity factor at the crack tip will increase. However, crack propagation only occurs in the event that stress intensity factor, $K_{I}$, is greater than the critical stress intensity factor, $K_{I C}$ (Zehnder 2012). Due to the existence of spatial disorder in the elastic energy release rate, crack initiation occurs within the material bulk due to lower fracture toughness values compared to the notch tip.

At relatively low disorder strengths and low notch widths of $64 \mu \mathrm{m}$ or less, crack initiation occurs at the notch, but as the notch width $W$ increases, the location of crack nucleation will deviate from this behavior. Figure 6 shows the crack propagation behavior of disorder strength, $R_{G}=0.2$, with respect to the various crack widths of $16 \mu \mathrm{m}, 64 \mu \mathrm{m}, 0.25 \mathrm{~mm}$, and $0.5 \mathrm{~mm}$. The load case and disorder distributions for these samples were held constant across the simulations. However, there 


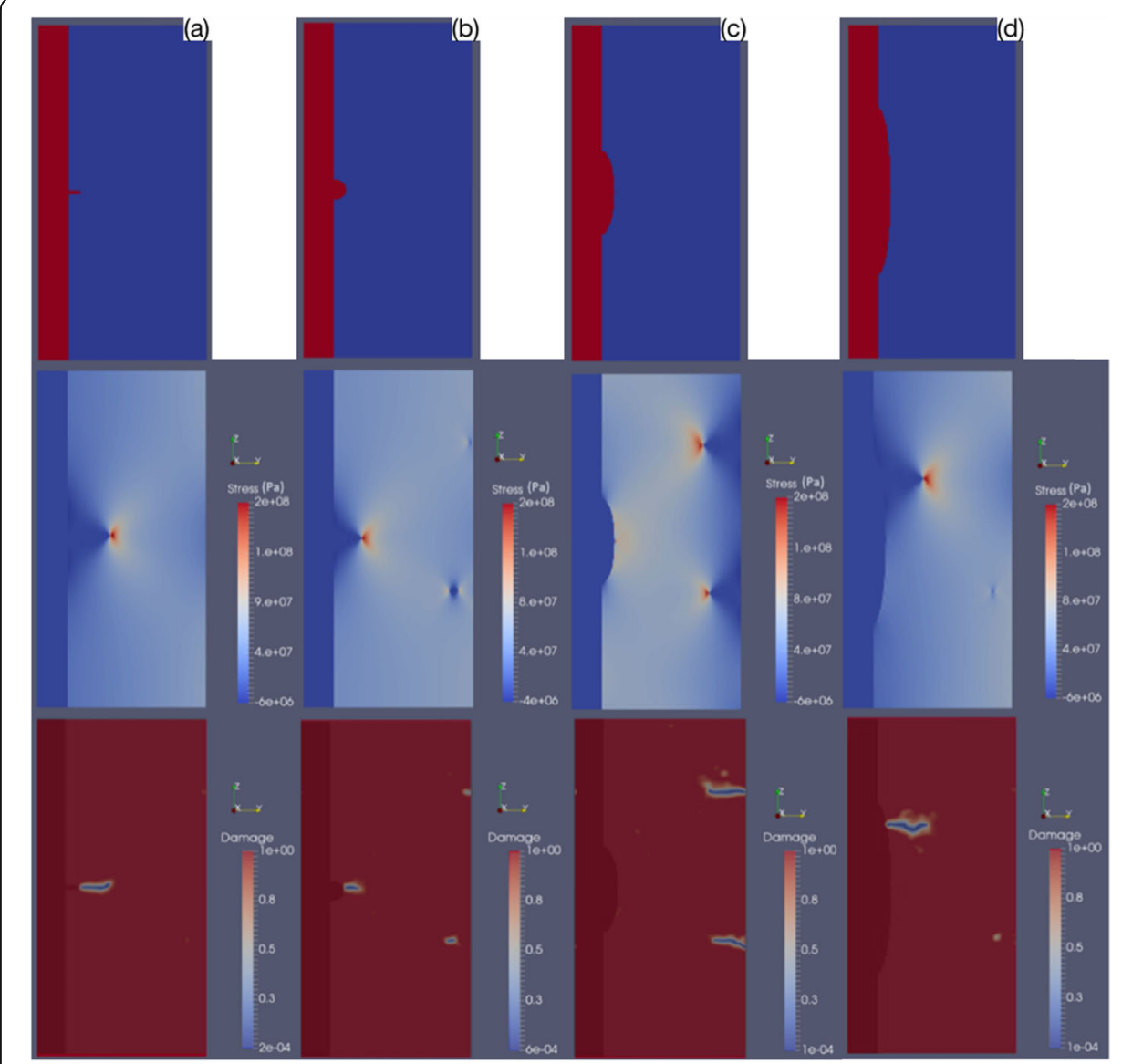

Fig. 6 Effect of notch width on crack initiation in a disordered crystal: Simulations of system size of $L_{x}=$ $8 \mu \mathrm{m}, \mathrm{L}_{\mathrm{y}}=0.5 \mathrm{~mm}$, and $\mathrm{L}_{z}=1.0 \mathrm{~mm}$ where $\mathrm{D}=2.995, \mathrm{R}_{\mathrm{G}}=0.2$, and (a) has a $\mathrm{W}$ of $16 \mu \mathrm{m}$ (b) has a $\mathrm{W}$ of $64 \mu \mathrm{m}(\mathbf{c})$ has a W of $0.25 \mathrm{~mm}$ (d) has a W of $0.5 \mathrm{~mm}$ where the texture, stress and damage distributions are displayed (top, middle, bottom)

is varying behavior in the crack nucleation location and, as a result, the crack propagation behavior. Upon examination of (c) and (d), the radius of curvature is much larger, allowing for lower stresses at the notch tip compared to the rest of the stresses present in the sample. Instead of crack initiation at the notch tip, nucleation occurs at a location where the critical strain energy release rate in the phase field energy distribution is statistically lower than the critical strain energy release rate at the notch tip. This behavior is observed across the same disorder strength with increasing notch width, and shows that there is a transition from brittle, notch-driven fracture to quasi-brittle, disorder-induced fracture. As a result, the initial notch width in the specimen can be concluded to have a characteristic effect on the crack initiation and propagation behavior.

\section{Average stress and damage through the transition from brittle to quasi-brittle behavior}

The transition in the fracture behavior is observed in terms of the average and maximum stresses, average damage, and crack initiation location. It is important to note that the stress at the notch tip, in the absence of disorder, is (Inglis, 1913): 


$$
\sigma_{\max }=\sigma_{\infty}(1+2 \sqrt{a / \rho})
$$

Where the stress at the tip of the notch, $\sigma_{\max }$, is a function of the applied stress, $\sigma_{\infty}$, the radius of curvature, $\rho$, and initial crack length, $a$. The applied stress is the tensile stress response of the sample as it is incrementally loaded at the specified strain rate. The radius of curvature, $\rho=b^{2} / a$, is a function of the initial crack length and the width of the notch, $W=2 b$, given that the minor axis of an ellipse is $2 b$. Inglis' solution expands on the previous work done by Kirsch where he analyzed the solution for a circular notch in the center of a specimen and found the stress around it to be proportionally $\sigma_{\max } \sim 3 \sigma_{\infty}$ (Kirsch 1898). Thus, the stress at the tip of the ellipse is proportional to the radius of curvature at the tip.

At the point of crack nucleation, there is an event in which the stress decreases sharply. This is, typically, described as an avalanche where a brittle crack begins to nucleate. In Fig. 7, at lower disorder strengths, the slope of each maximum stress curve is initially increasing steadily for each individual notch width. As disorder and notch width increase, this characteristic, indicative of brittle fracture, begins to soften and disappear. Instead, it is replaced by a sharp increase in maximum stress profile as the crack nucleates. Furthermore, after the crack nucleates, there are stochastic events which create avalanches. As deformation continues, the maximum stress continues to obtain high values at the crack tip. Once the crack tip develops, the radius of curvature at the tip becomes very sharp, leading to very high stresses. Due to the discretization unit, a minimum radius of curvature is allowed for the crack tip in the simulation. As
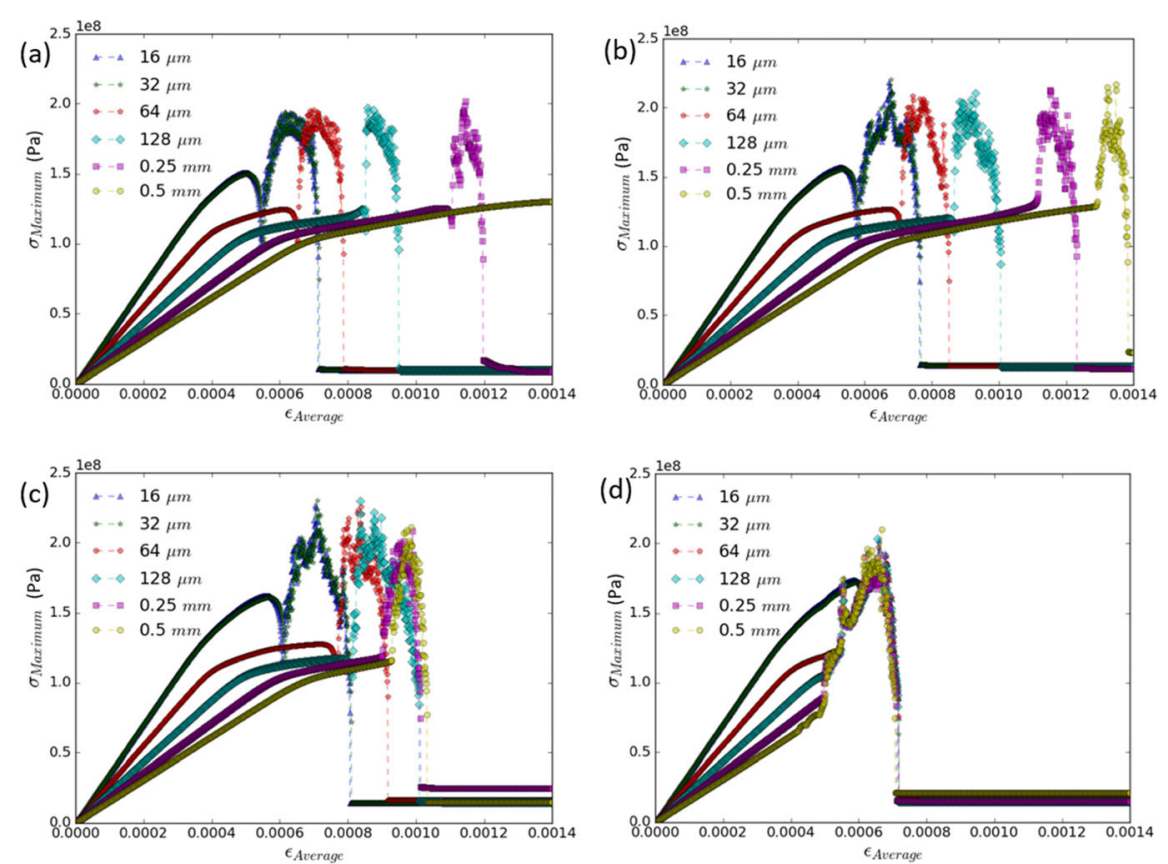

Fig. 7 Effect of disorder on maximum stress along loading z-direction: The maximum stress of samples of $L_{x}=8 \mu \mathrm{m}, L_{y}=0.5 \mathrm{~mm}$, and $L_{z}=1.0 \mathrm{~mm}$ where $D=2.995$ as a function of the average strain with respect to $R_{G}$ where (a) has a $R_{G}=0.0$, (b) has a $R_{G}=0.1$, (c) has a $R_{G}=0.2$, and (d) has a $R_{G}=0.4$. Naturally, there is observed stochasticity due to the fact that maximum stress reflects the disorder distribution (Alava et al. 2009). The figure legends signify the notch width $W$ that varies from 0.25 to $128 \mu \mathrm{m}$ 
the crack propagates, the discretized unit ahead of the crack tip experiences the greatest increase in stress and quickly satisfies criterion for damage in the phase field. Once the crack is initiated and begins to propagate, the specimens for a single disorder strength obtain similar peak values for the fractured tip. As seen in the stress progression of Fig. 5, once the crack nucleates, the stress profile concentrates at the tip of the crack. After complete failure, the maximum stress decreases sharply to a residual value. At higher disorder strengths, the maximum stress curves fail to exhibit any of the same trends that the lower disorder strength curves do. The maximum stress values across all notch widths at $R_{G}=0.2$ and $R_{G}=0.4$ show larger variation in the peak maximum stress values not consistent with the lower disorder strengths. Furthermore, the initial maximum stress decrease at crack initiation dampens out as notch width and disorder strength increase.

At lower disorder strengths, the average stress follows the typical brittle fracture regime with no avalanches or fluctuation in the decreasing average stress. Also, the average stress required for fracture increases as the notch becomes shallow (see Fig. 8): Due to the decreasing curvature at the notch tip, the sample requires a larger applied tensile load for damage to occur. At higher disorder strengths, the average stress enters this quasi-brittle fracture regime where there are avalanches as the sample reaches its peak average stress and crack nucleation occurs. Furthermore, this regime, also, produces fluctuations as crack propagation occurs. We focus on the behavior near this brittlequasi-brittle transition.

Overall, the crack initiates at the notch tip when the local critical strain energy release rate is reached and average stress begins to decrease. The average stress curve's response to this event is that the stress decreases relatively quickly, giving brittle

(a)
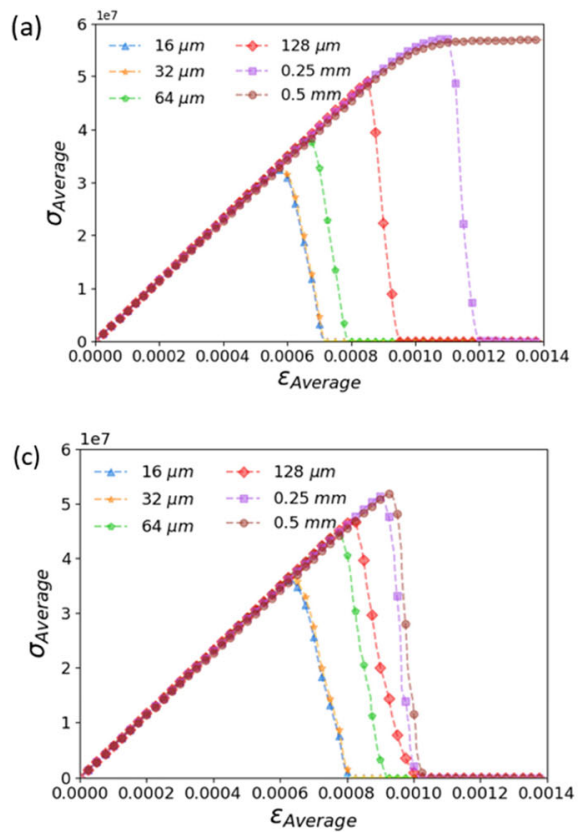

(b)

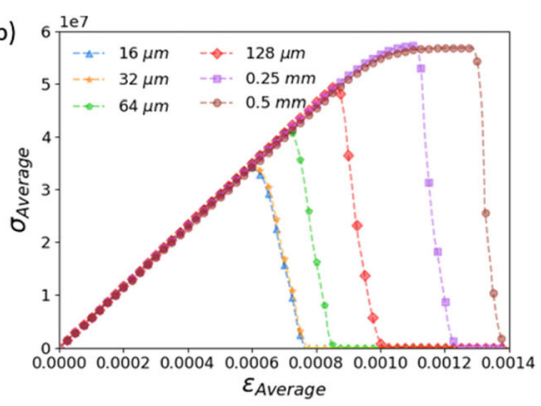

(d)

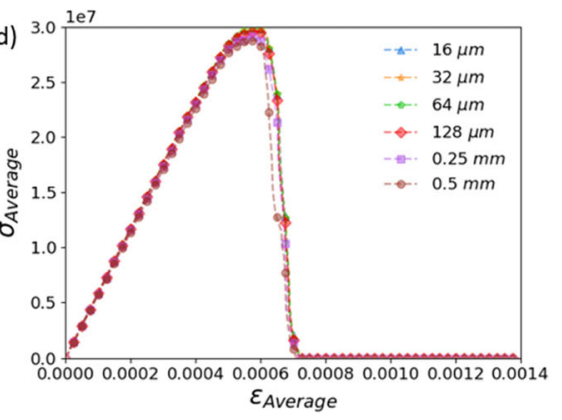

Fig. 8 Effect of disorder on average stress: The average stress of the specimens of $L x=8 \mu m, L y=0.5 \mathrm{~mm}$, and $L z=1.0$. $\mathrm{mm}$ where $D=2.995$ as a function of the average strain with respect to $R G$ where (a) has a $R G=0.0$, (b) has a $R G=0.1$, (c). has a $R G=0.2$, and (d) has a $R G=0.4$. The figure legends signify the notch width $\mathrm{W}$ that varies from 0.25 to $128 \mu \mathrm{m}$ 
signatures. In Fig. 8, the behavior of the samples can be characterized as brittle when observing the average stress curves at the lower disorder strengths from (a) and (b). At lower disorder strengths, the sample obtains a small, residual average stress value after complete failure and follows the expected trend: As notch width increases, the maximum average stress increases before crack initiation occurs at the notch tip. With the increasing radius of curvature, the sample exhibits a plastic characteristic: As the strain is increased, there is no increase with respect to the average stress. However, as the disorder strength increases, the maximum average stress values decrease for higher crack widths and crack initiation occurs earlier in the load case. The fluctuation at which this response occurs is of importance to classifying the fracture behavior. Furthermore, the curves appear to collapse on each other, displaying similar quasi-brittle fracture behavior. Through these identifications, and taking into account all evidence compiled in our work for all studied parameter combinations, we can infer a phase diagram for the regime where quasi-brittle fracture emerges (see Fig. 9).

In addition, the event size of fracture displays a characteristic effect that indicates brittle or quasi-brittle fracture. The measured event size for a brittle sample exhibits a single large stress drop where failure occurs. However, samples that experience quasibrittle fracture often exhibit multiple stress drops. Therefore, we consider not only the stress event size, but the number of events with their corresponding stress drops.

As the disorder ratio increases, the average stress curves transition from brittle to quasi-brittle fracture, as expected. Figure 10 shows the characteristic difference between brittle and quasi-brittle samples: Brittle samples with low disorder only have a single stress event during fracture where stress events are defined as significant stress drops in the average stress profile. In contrast, quasi-brittle samples are seen to have multiple stress events in Fig. 10a. Though one stress event dominates fracture, the precursor events still have significant stress drops that must be considered. Furthermore, in Fig. 10b, the stress events demonstrate clear insensitivity in the average maximum stress at similar strain values.

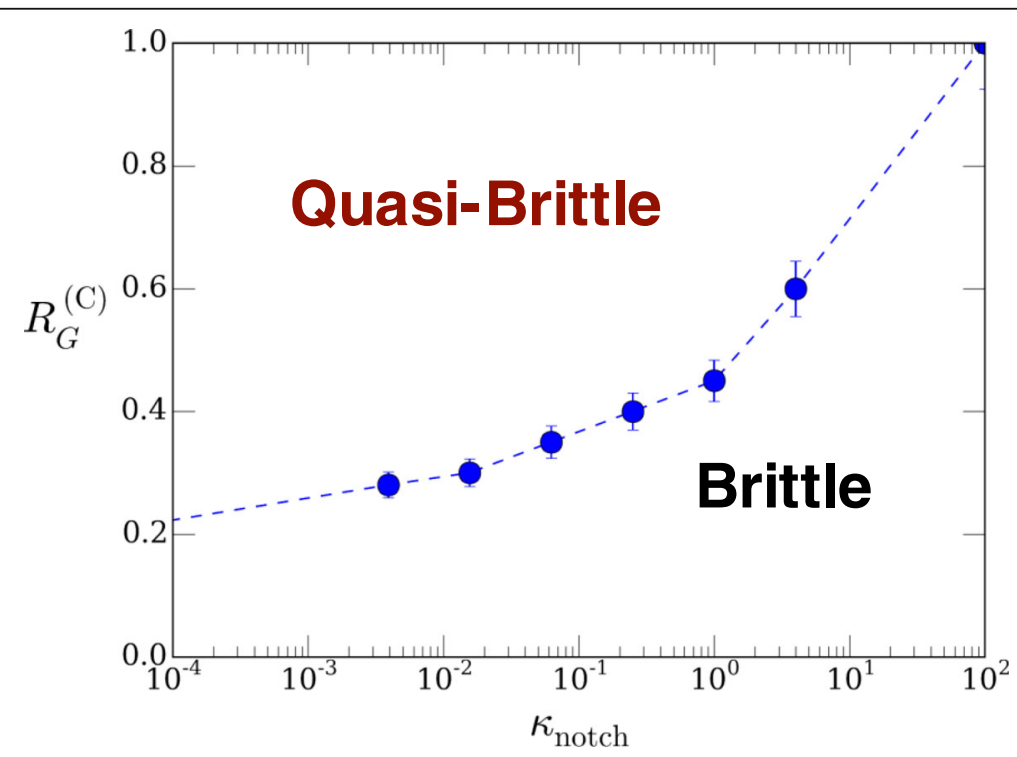

Fig. 9 Phase diagram of the studied model 

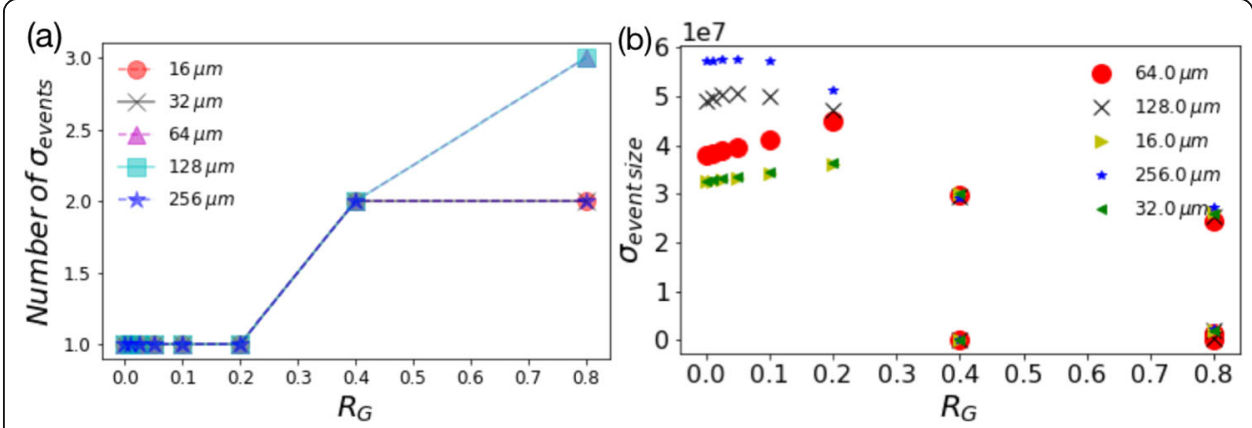

Fig. 10 Significant Stress Events for Brittle and Quasi-brittle Fracture: The number and size of the stress events were measured as a function of RG where (a) is a series of histogram curves for various $W$ and (b) is a plot of the event size for various $W$. The figure legends signify the notch width $W$ that varies from 16 to $256 \mu \mathrm{m}$

Characterizing the effect of increasing the critical strain energy release rate variance across several system sizes is necessary to understanding how fracture behavior transition is impacted by the sample length, $L_{y}$. One way to indicate this is by the difference in the maximum strain, average strain or other observables at fracture, between the very sharp $(W=16 \mu \mathrm{m})$ and very shallow notch $(W=0.5 \mathrm{~mm})$ cases. This difference can be viewed as an order parameter for the aforementioned phase transition. As shown in Fig. 11 for various notch widths, the average stress curves begin to collapse on each other as the disorder strength increases. Data was collected for three system sizes, ranging from $L_{y}$ of 0.25 to $1.0 \mathrm{~mm}$. Also, disorder strengths were tested for the range 0.0 to 0.8 .

As we observed the behavior of the average stress curves (Fig. 8), once the maximum average stress is reached the stress begins to decrease relatively linearly. However, the stress does not obtain a value of zero rather a residual stress value. Figure 11a shows the difference in average strain of the notch width $W=16 \mu \mathrm{m}$ and $0.5 \mathrm{~mm}$ at the location of which the residual stress was present. In panel (b) for both cases of $L_{y}=0.25$ and $1.0 \mathrm{~mm}$, the average stress curve of notch widths $16 \mu \mathrm{m}$ and $0.5 \mathrm{~mm}$ are very similar in behavior and magnitude, collapsing on each other at approximately same disorder strength. The behavior of these curves exhibit a much shaper decline than $L_{y}=0.5 \mathrm{~mm}$ in the maximum difference of average stress for the notch widths. The differences are expected for the fact that disorder is not sample averaged.
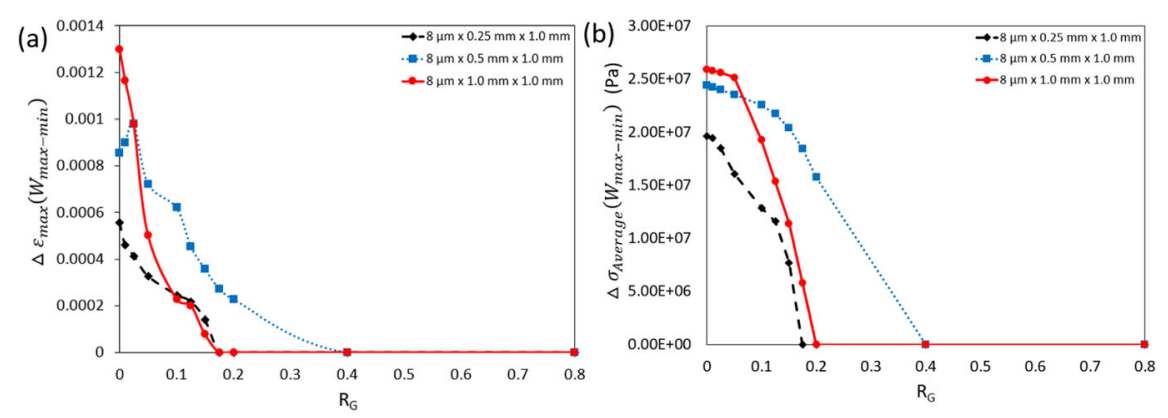

Fig. 11 Difference in spatially maximum strain and average stress as a function of disorder strength and maximum and minimum crack width: (a) the difference in the maximum strain of the notch width of $16 \mu \mathrm{m}$ as a function of disorder (b) the difference in the peak average stress of the notch width of $0.5 \mathrm{~mm}$ as a function of disorder 
In Fig. 12, the average damage is plotted as a function of the average strain. The disorder strength increases from 0.0 to 0.4 . At lower disorder strengths, the behavior is consistent with the observations made in regards to the average stress where it can be characterized as brittle fracture behavior. Also, at relatively low disorder strengths before the transition, the average damage increases as the notch width increases and the crack initiation occurs at a larger strain. The relationship between maximum achieved average damage and the crack width may be described by considering that the strain value at the crack nucleation for higher crack widths is greater than those in the lower crack width's crack nucleation strain values. For lower disorder strengths, where a single crack initiates at the notch tip, the average damage corresponds proportionally to the crack length as no other damage occurs but at the crack tip, as it propagates.

Figure 13 shows the plots of the dimensionless parameter for the rate of change of the average stress (namely $\frac{\sqrt{\rho} \Delta \sigma_{z z}}{K_{I C}}$ with $\rho$ the notch radius of curvature) versus incremental step. These plots may be viewed as avalanche timeseries, analogously to other complex systems (Papanikolaou et al. 2017). (Here, the critical stress intensity factor can be defined as function of the maximum average stress, $\sigma_{\infty}$, maximum , and the crack length, a: $\left.K_{I C}=\sigma_{\infty, \text { maximum }} \sqrt{\pi a}\right)$.

Since the simulations are displacement controlled, the behavior of the plots remains unaltered when considering an increment of the average strain across the sample. Fundamentally, the derivative of the average stress plots would show a constant value, initially, as the constant slope of elastic stress remains consistent with the increasing load case. However, after complete failure, the rate of change in the average stress exhibited across all simulations is large. The sharp increase in the rate of change of average stress
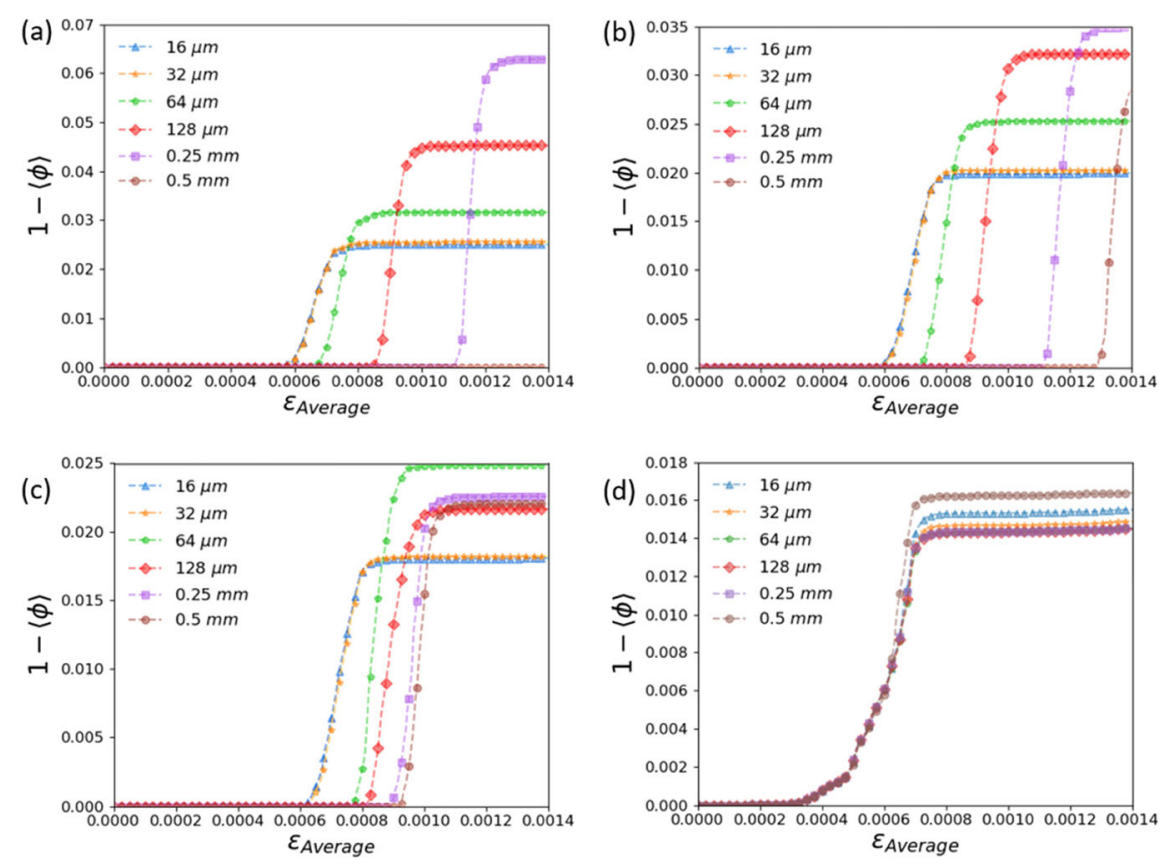

Fig. 12 Effect of disorder on average damage: The average damage of the specimens of $L_{x}=8 \mu m, L_{y}=0.5$ $\mathrm{mm}$, and $L_{z}=1.0 \mathrm{~mm}$ where $D=3.995$ as a function of the average strain with respect to $R_{G}$ where (a) has a $R_{G}=0.0$, (b) has a $R_{G}=0.1$, (c) has a $R_{G}=0.2$, and (d) has a $R_{G}=0.4$. The figure legends signify the notch width $\mathrm{W}$ that varies from 0.25 to $128 \mu \mathrm{m}$ 

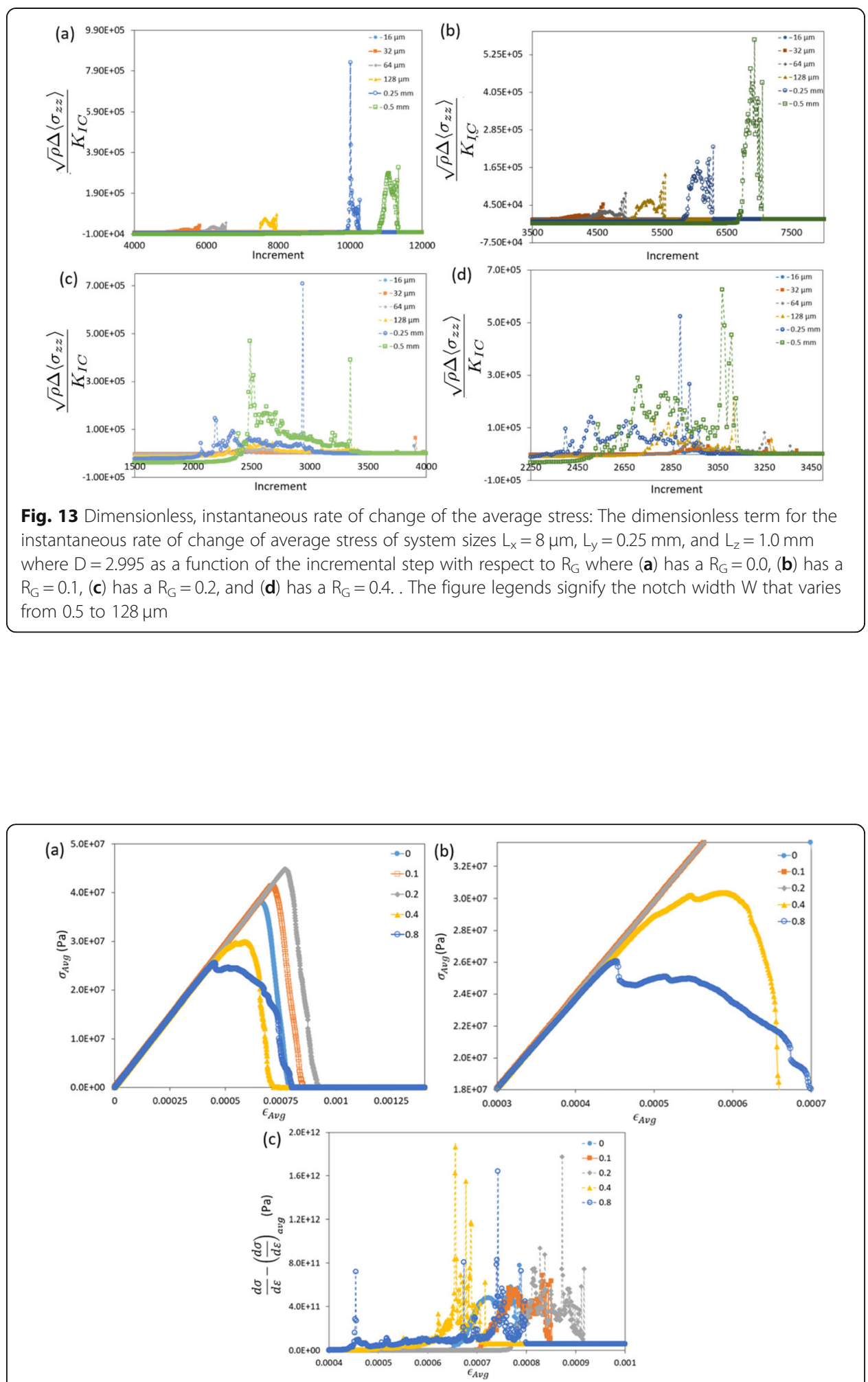

Fig. 14 Quantification of the fluctuations at crack nucleation and growth of average stress: Simulations of sample size $L_{x}=8 \mu \mathrm{m}, L_{y}=0.5 \mathrm{~mm}$, and $L_{z}=1.0 \mathrm{~mm}$ where $D=2.995$ and $W=64 \mu \mathrm{m}$ as a function of the average strain where (a) is the average stress vs. average strain, (b) is the zoomed-in view of the fluctuations for disorder strengths 0.4 and 0.8 , and (c) is the difference in the rate of change of the average stress and the initial rate of change of linear increase in average stress vs. average strain. . The figure legends signify the disorder variance ratio $R_{G}$ that varies from 0.0 to 0.8 
occurs at about a magnitude of $10^{2} \mathrm{kPa}$ before the parameter becomes dimensionless. This observation indicates that the crack has fully propagated through the sample and the average stress has dropped to values at which it is considered the residual stress. As the disorder strength increases, the event of complete failure across all crack widths begins earlier in the load case. In panel (a), the rate of change in average stress appears to exhibit the same general trend as the average stress curve. As the disorder strength is increased, the change in the average stress begins to exhibit the same stochastic behavior of the respective disorder strengths. These abrupt crack-initiation events correspond to avalanche precursors to crack initiation in the model we investigated. The precursors become larger and more intricate as $R_{G}$ increases, and may amount to 1-5 MPa for the model material (c.f. Figure 14), observable range in typical experimental set-ups (Ritchie and Peters 2001).

\section{Conclusions}

A model material is developed to obtain a better understanding of the realistic response that a structurally disordered specimen would undergo in mode I fracture loading conditions. Manufacturing and processing promote such structural disorder fluctuations in material properties at the microscale. The Weierstrass-Mandelbrot function was implemented in a material simulation software (Roters et al. 2019), towards the investigation of crack initiation in disordered microstructures that may deform elastically and plastically. For brittle fracture, the theory of fracture mechanics expects that crack initiation would occur at the notch tip and this behavior is, also, exhibited at lower disorder strengths where the critical strain energy release rate variance is not large enough to have an effect on the initial crack nucleation location. However, at higher disorder strengths, the critical strain energy release rate variance is large enough to induce crack nucleation on the bulk sample where stresses are higher than those present at the notch tip. The purpose of increasing the disorder strength in the material simulations was to identify the transition of brittle to quasi-brittle fracture behavior at a metal's microscale.

In this work, we showed that there is a relationship of the fracture behavior between notch-driven (geometry-based) and disorder-driven (material-based) fracture where it transitions from brittle to quasi-brittle fracture behaviors for crack nucleation and propagation. These simulations demonstrate that there are observable avalanche precursors that could be tracked in experimental efforts through well controlled experimental setups by regulating the geometry of a notch. It is plausible that the magnitude of these avalanche precursors extend into a non-trivial scaling function that can be used throughout the critical regime; if that's the case, as this work suggests, then it should become possible to utilize these events towards safe and non-invasive prediction of crack initiation in intermetallics and other disordered brittle materials.

\section{Supplementary information}

Supplementary information accompanies this paper at https://doi.org/10.1186/s41313-019-0017-0.

Additional file 1: Supplementary Information. Brittle to Quasi-Brittle Transition and Crack Initiation Precursors in Crystals with Structural Inhomogeneities. 


\section{Acknowledgements}

We would like to thank M. Alava, C. Reichhardt, H. Song, and E. Van der Giessen for helpful and inspiring comments on this manuscript. We would also like to thank J. El Awady and K. Hemker for inspiring discussions in the beginning of this project. We would like to acknowledge the use of the WVU High Performance Computing facilities for this project.

\section{Authors' contributions}

CW, SP had the original insight towards this work. PS, FR, SP modified the original DAMASK code towards the purposes of the work. SP produced the simulation data presented in the manuscript. SP, JT analyzed and interpreted the data. All authors read and approved the final manuscript.

\section{Funding}

We would like to acknowledge support from NSF, DMR-MPS, Award No\#1709568 "Crack growth during fatigue in Ni superalloys: Physical origin of stochastic jumps and their predictive role using statistical approaches".

\section{Availability of data and materials}

The data sets used and/or analyzed during the current study are available from the corresponding author on reasonable request.

\section{Competing interests}

The authors declare that they have no competing interests.

\section{Author details}

'Department of Mechanical \& Aerospace Engineering, West Virginia University, Morgantown, WV 26505, USA

${ }^{2}$ Department of Physics \& Astronomy, West Virginia University, Morgantown, WV 26505, USA. ${ }^{3}$ Max-Planck-Institut für Eisenforschung, Max-Planck-Straße 1, 40237 Düsseldorf, Germany. ${ }^{4}$ Materials and Manufacturing Directorate, Air Force Research Laboratory, Wright Patterson Air Force Base, Dayton, OH 45433-7817, USA.

Received: 18 April 2019 Accepted: 23 September 2019

Published online: 01 November 2019

\section{References}

M.J. Alava, P.K. Nukala, S. Zapperi, Role of disorder in the size scaling of material strength. Phys. Rev. Lett. 100(5), 055502 (2008)

M.J. Alava, P.K.V.V. Nukala, S. Zapperi, Size effects in statistical fracture. J. Phys. D. Appl. Phys. 42, 214012 (2009)

I.S. Aranson, V.A. Kalatsky, V.M. Vinokur, Continuum field description of crack propagation. Phys. Rev. Lett. 85(1), 118 (2000)

R.J. Asaro, Crystal plasticity. J. Appl. Mech. 50(4b), 921-934 (1983)

Asaro, R. J, Lubarda V.. Mechanics of Solids and Materials. Cambridge University Press, 2006

Z.P. Bažant, Size effect in blunt fracture: Concrete, rock, metal. J. Eng. Mech. 110(4), 518-535 (1984)

Z.P. Bažant, Fracture in Concrete and Reinforced Concrete (1985)

Z.P. Bažant, Size effect aspects of measurement of fracture characteristics of quasi-brittle material. Adv. Cem. Based Mater. 4(3-4), 128-137 (1996)

Z.P. Bažant, J.-L. Le, M.Z. Bazant, Scaling of strength and lifetime probability distributions of Quasi-brittle structures based on atomistic fracture mechanics. Proc. Natl. Acad. Sci. 106(28), 11484-11489 (2009)

Z.P. Bažant, Z. Li, Zero-Brittleness Size-Effect Method for One-Size Fracture Test of Concrete. J. Eng. Mech. 122(5), 458-468 (1996). https://doi.org/10.1061/(ASCE)0733-9399(1996)122:5(458)

Z.P. Bažant, B.H. Oh, Crack band theory for fracture of concrete. Mater. Constr. 16(3), 155-177 (1983)

Z.P. Bažant, P.A. Pfeiffer, in Proceedings of the second symposium on interaction of non-nuclear munition with structures, Florida, USA. Test of shear fracture and strain-softening in concrete (1985), pp. 254-264

Z.P. Bažant, J. Planas, Fracture and Size Effect in Concrete and Other Quasi-Brittle Structures (1998)

Z.P. Bažant, M.R. Tabbara, M.T. Kazemi, G. Pijaudier-Cabot, Random particle model for fracture of aggregate or fiber composites. J. Eng. Mech. 116(8), 1686-1705 (1990)

Z.P. Bažant, Q. Yu, Size effect in fracture of concrete specimens and structures: New problems and progress. Acta. Polytechnica. 44(5-6) (2004)

J.P. Campbell, R.O. Ritchie, K.T.V. Rao, The effect of microstructure on fracture toughness and fatigue crack growth behavior in Y-titanium aluminide based intermetallics. Metallurgical Mater. Trans. A 30(3), 563-577 (1999)

L.R. Carney, J.J. Mecholsky, Relationship between fracture toughness and fracture surface fractal dimension in AISI 4340 steel. Mater. Sci. Appl. 4(04), 258 (2013)

A. Carpinteri, Stress-singularity and generalized fracture toughness at the vertex of re-entrant corners. Eng. Fract. Mech. 26, 143-155 (1987)

A. Carpinteri, R. Brighenti, A. Spagnoli, Size effect in beams with rounded-tip V-notch. Mater. Sci. 32(3), 325 (1996)

H. Clemens, S. Mayer, Design, processing, microstructure, properties, and applications of advanced intermetallic TiAl alloys. Adv. Eng. Mater. 15(4), 191-215 (2013)

D.M. Dimiduk, Gamma titanium aluminide alloys_An assessment within the competition of aerospace structural materials. Mater. Sci. Eng. A 263(2), 281-288 (1999)

M.L. Dunn, W. Suwito, S.J. Cunningham, Stress Intensities at notch singularities. Eng. Fracture Mech. 57(4), 417-430 (1997a)

M.L. Dunn, W. Suwito, S.J. Cunningham, Fracture initiation at sharp notches: correlation using critical stress intensities. Int. J. Solids Structures 34(29), 3873-3883 (1997b)

F.G. Evans, in Charles C. Thomas Publisher. Mechanical properties of bone. 881 (1973)

D.L. Grote, S.W. Park, M. Zhou, Dynamic behavior of concrete at high strain rates and pressures: I. experimental characterization. Int. J. Impact Eng. 25(9), 869-886 (2001) 
J. Han, S. Xiao, J. Tian, Y. Chen, L. Xu, X. Wang, Y. Jia, Z. Du, S. Cao, Grain refinement by trace TiB2 addition in conventional cast TiAl-based alloy. Mater. Charact. 106, 112-122 (2015)

E.B. Herbold, J.B. Jordan, N.N. Thadhani, Effects of processing and powder size on microstructure and reactivity in arrested reactive milled Al+ Ni. Acta Mater. 59(17), 6717-6728 (2011)

D. Hu, Effect of composition on grain refinement in TiAl-based alloys. Intermetallics 9(12), 1037-1043 (2001)

C.E. Inglis, "Stresses in a plate due to the presence of cracks and sharp corners." Trans Inst Naval Archit. 55, 219-241 (1913)

Y.-W. Kim, Ordered intermetallic alloys, part III: Gamma titanium aluminides. JOM J. Miner. Met. Mater. Soc. 46(7), 30-39 (1994)

Y.-W. Kim, D.M. Dimiduk, Progress in the understanding of gamma titanium aluminides. JOM J. Miner. Met. Mater. Soc. 43(8), 40-47 (1991)

C. Kirsch, "Die theorie der elastizitat und die bedurfnisse der festigkeitslehre." Zeitschrift des Vereines Deutscher Ingenieure. 42, 797-807 (1898)

Lapin, J. "TiAl-based alloys: Present status and future perspectives." Conference proceedings METAL, vol. 19, 21.5, (2009)

S. Lay, A.-R. Yavari, Evidence for disordered character of grain boundaries in a Ni3Al-based alloy during reordering. Acta Mater. 44(1), 35-41 (1996)

Mandelbrot, B. B. "Fractals: form, chance and dimension." Fractals: form, chance and dimension., by Mandelbrot, BB. San Francisco (CA, USA): WH Freeman \& Co., 16+ 365 p. (1979)

B.B. Mandelbrot, D.E. Passoja, A.J. Paullay, Fractal character of fracture surfaces of metals. Nature 308(5961), 721 (1984)

S. Ochiai, S. Kuboshima, K. Morishita, H. Okuda, T. Inoue, Fracture toughness of Al 2 O 3 fibers with an artificial notch introduced by a focused-ion-beam. J. Eur. Ceramic Soc. 30(7), 1659-1667 (2010)

S. Papanikolaou, C. Yinan, N. Ghoniem, Avalanches and plastic flow in crystal plasticity: an overview. Model. Simul. Mater. Sci. Eng. 26(1), 013001 (2017)

R.O. Ritchie, J.O. Peters, Small fatigue cracks: Mechanics, mechanisms and engineering applications. Mater. Trans. 42(1), 58-67 (2001)

F. Roters, M. Diehl, P. Shanthraj, P. Eisenlohr, C. Reuber, S.L. Wong, T. Maiti, A. Ebrahimi, T. Hochrainer, H.O. Fabritius, S. Nikolov, DAMASK-The Düsseldorf Advanced Material Simulation Kit for modeling multi-physics crystal plasticity, thermal, and damage phenomena from the single crystal up to the component scale. Comput. Mater. Sci. 158, 420-478 (2019)

E. Schlangen, J. Van Mier, Experimental and numerical analysis of micromechanisms of fracture of cement-based composites. Cement Concrete Composites 14(2), 105-118 (1992)

E. Schlangen, E.J. Garboczi, Fracture simulations of concrete using lattice models: computational aspects. Eng. Fracture Mech. 57(2), 319-332 (1997)

H.E.J.G. Schlangen, Experimental and numerical analysis of fracture processes in concrete. HERON 38(2), 1993 (1993)

P. Shanthraj, O. Rezvanian, M.A. Zikry, Electrothermomechanical finite-element modeling of metal microcontacts in MEMS. J. Microelectromech. Syst. 20(2), 371-382 (2011)

P. Shanthraj, L. Sharma, B. Svendsen, F. Roters, D. Raabe, A phase field model for damage in elasto-viscoplastic materials. Comput. Methods Appl. Mech. Eng. 312, 167-185 (2016)

L. Skarynski, J. Tejchman, Calculations of fracture process zones on Meso-scale in notched concrete beams subjected to three-point bending. Eur. J. Mech. A/Solids 29, 746-760 (2010). https://doi.org/10.1016/j.euromechsol.2010.02.008

N.S. Stoloff, C.T. Liu, S.C. Deevi, Emerging applications of intermetallics. Intermetallics 8(9), 1313-1320 (2000)

Y. Tian, D. McAllister, H. Colijn, M. Mills, D. Farson, M. Nordin, S. Babu, Rationalization of microstructure heterogeneity in Inconel 718 builds made by the direct laser additive manufacturing process. Metall. Mater. Trans. A 45(10), 4470-4483 (2014)

I. Tikhovskiy, D. Raabe, F. Roters, Simulation of earing during deep drawing of an Al-3\% mg alloy (AA 5754) using a texture component crystal plasticity FEM. J. Mater. Process. Technol. 183(2), 169-175 (2007)

A.A. Tseng, Recent developments in micromilling using focused ion beam technology. J. Micromech. Microeng. 14(4), R15 (2004)

S.K. Vajpai, K. Ameyama, A novel powder metallurgy processing approach to prepare fine-grained Ti-rich TiAl-based alloys from pre-alloyed powders. Intermetallics 42, 146-155 (2013)

P.F. Walsh, Fracture of plain concrete. Indian Concrete J. 46(11) (1972)

L. Wang, J. Shen, Y. Zhang, H. Fu, Microstructure, fracture toughness and compressive property of as-cast and directionally solidified NiAl-based eutectic composite. Mater. Sci. Eng. A 664, 188-194 (2016)

K. Weierstrass, Uber continuierliche Functionen eines reellen Argumentes, die für keinen Werth des lezteren einen bestimmten Differentialquotient besitzen (Math, Werke II, 1895)

M.L. Williams, Stress singularities resulting from various boundary conditions in angular corners of plates in extension. J. Appl. Mech. 74, 526-528 (1952)

A.T. Zehnder, Fracture Mechanics (Lecture Notes in Applied and Computational Mechanics) (2012)

L. Zhu, Z.F. Xu, P. Liu, Y.F. Gu, Effect of processing parameters on microstructure of laser solid forming Inconel 718 superalloy. Opt. Laser Technol. 98, 409-415 (2018)

\section{Publisher's Note}

Springer Nature remains neutral with regard to jurisdictional claims in published maps and institutional affiliations. 